\title{
Rehabilitación y Trabajo Social en centros de privación de libertad en Ecuador.
}

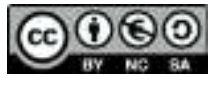

\section{Rehabilitation and Social Work in detention centers in Ecuador.}

\author{
Silvia Jovanna Altamirano Altamirano ${ }^{1}$
}

Recibido: 10-02-2019 / Revisado: 15-02-2019 /Aceptado: 04-03-2019/ Publicado: 14-06-2019

\begin{abstract}
DOI: $\underline{\text { https://doi.org/10.33262/cienciadigital.v9i2.383 }}$

The management of Social Work in the processes of social rehabilitation is an urgent need in the centers of deprivation of liberty for adults in conflict with the Law in order to contribute to retake their family bond, reduce recidivism and may be productive entities for the society. So this research focuses on knowing how it is happening and what characteristics are associated with Rehabilitation, as well as how to understand the Social Work intervention. For this purpose, 198 prisoners were deprived of freedom in the city of Riobamba (south central area of Ecuador). The results obtained determined that in this penitentiary institution there are no intervention activities of Social Work due to the lack of specialized professionals in the area, this being a factor that affects the effective processes of family reintegration, it was also possible to verify that there are no plans of intervention in which the PPLs are participating. It is concluded analyzing the subject and at the same time it is proposed to design an intervention plan for Social Work to improve the processes of family reintegration as a basic tool in this institution, through strategies that strengthen family ties to achieve an effective integration of the PPL with your family once freedom is restored.
\end{abstract}

Keywords: reintegration, family, intervention, recidivism, centers of deprivation of liberty.

\section{Resumen}

La gestión del Trabajo Social en los procesos de rehabilitación social es una necesidad impostergable en los centros de privación de libertad para personas adultas en conflicto con la Ley con el fin de contribuir a retomar su vínculo familiar, disminuya la reincidencia y puedan ser entes productivos para la sociedad. Por lo que esta investigación se centra en conocer cómo está ocurriendo y qué características se asocian con la Rehabilitación, así como la manera de

${ }^{1}$ Universidad Técnica de Ambato, Facultad de Jurisprudencia y Ciencias Sociales, Ambato, Ecuador, saltamirano67@yahoo.com 
entender la intervención Trabajo Social. Para ello se encuestó a 198 privados de la libertad de la ciudad de Riobamba (zona centro sur del Ecuador). Los resultados obtenidos determinaron que en esta institución penitenciaria no se realizan actividades de intervención de Trabajo Social debido a la falta de profesionales especializados en el área, siendo éste un factor que afecta los procesos efectivos de reinserción familiar, además se pudo comprobar que no existen planes de intervención en los cuales se encuentren participando los PPLs. Se concluye analizando el tema y a la vez se propone diseñar un plan de intervención de Trabajo Social para mejorar los procesos de reinserción familiar como una herramienta básica en esta institución, a través de estrategias que fortalezcan los vínculos familiares para lograr una efectiva integración del PPL con su familia una vez recuperada la libertad.

Palabras clave: reinserción, familia, intervención, reincidencia, centros de privación de la libertad.

\section{Introducción.}

El Trabajo Social en los centros de privación de la libertad juega un papel importante dentro de los ámbitos de actuación profesional pues trata de modificar las conductas, crear hábitos y valores positivos en los PPra logar una convivencia sana y saludable en el interior de estos centros y en beneficio de la sociedad y familia; de ahí la necesidad de que el profesional del trabajo social logre alcanzar la reinserción familiar del individuo con el fin que éste tome conocimiento e identifique las circunstancias que originaron conducta delictiva y las consecuencias derivadas de la privación de la libertad.

En el Ecuador la presencia del profesional de Trabajo Social en los centros penitenciarios es reducido y no guarda coherencia con el número de reclusos; actualmente existen alrededor de 36 mil personas privadas de libertad, distribuidas en 49 centros de privación de libertad; 29 son centros de Rehabilitación Social (CRS) y 20 centros de detención provisional (CDP), adicionalmente existen 3 casas de confianza en las provincias de Pichincha, Guayas y Napo. La tasa es de 147 personas privadas de libertad por cada 100 mil habitantes, existiendo una sobrepoblación del $42 \%$ en los diferentes Centros de Rehabilitación Social (Palma, 2017). La población penitenciaria tiene cifras del 91.9\% los cuales son varones y el $8.10 \%$ son mujeres, dando a notar que en nuestro país hay más personas privadas de la libertad varones que mujeres. (Álvarez, y otros, 2014).

Esta estadística se incrementa permanentemente con el pasar de los años en lugar de disminuir debido a la reincidencia (50\%), cifras que van en aumento por un lado y por otro, la ausencia de procesos adecuados de intervención social ocasiona la desmotivación personal hacia la participación en programas de rehabilitación existentes, el ambiente carcelario afecta el estado psicológico y social del recluso, incentivando resentimientos familiares y sociales que dificultan una efectiva reinserción, por ello, el objetivo general es determinar la incidencia de la intervención del trabajo social en los procesos de reinserción familiar en el Centro de Privación de Libertad para personas adultas en 
conflictos con la Ley en la ciudad de Riobamba (CPLPACLR).

\section{Metodología.}

El presente trabajo sobre la intervención del Trabajo Social y los procesos de reinserción familiar en el Centro de Privación de la Libertad para Personas Adultas en Conflicto con la Ley Riobamba, es de carácter descriptivo, correlacional bajo en enfoque cualicuantitativo. La técnica utilizada para la recolección de información fue la encuesta a través de un cuestionario con 16 preguntas de alternativa múltiple y opción cerrada, los datos fueron procesados, tabulados, graficados para el respectivo análisis e interpretación de resultados.

\section{Resultados.}

A continuación, se muestran los datos descriptivos en porcentajes respecto a la frecuencia de entrevista con Trabajador Social (tabla 1), a la participación en programas de rehabilitación (tabla 2), recibe orientación psicosocial (tabla 3), frecuencia de recepción de visitas (tabla 4), tipo de relación con su familia (tabla 5), se encuentra en proceso de reinserción familiar (tabla 6), es visitado por amigos (tabla 7), la familia tienen conocimiento donde se encuentra (tabla 8), al cumplir sentencia, su familia lo espera (tabla 9), tiene algún proyecto de vida (tabla 10), y es usted reincidente (tabla 11).

Tabla 1: Frecuencia de entrevista con Trabajador Social

\begin{tabular}{|c|c|c|c|c|}
\hline Alternativa & Frecuencia & Porcentaje & Porcentaje válido & Porcentaje acumulado \\
\hline Casi siempre & 8 & 4 & 4 & 4 \\
\hline Nunca & 188 & 94,9 & 94,9 & 99 \\
\hline Siempre & 2 & 1 & 1 & 100 \\
\hline Total & 198 & 100 & 100 & \\
\hline
\end{tabular}

La mayoría de los reclusos nunca han tenido entrevistas con un profesional de Trabajo Social porque el CPLPACL Riobamba carece de un experto en esta área para que lo oriente y ayude a conocer cómo está su situación legal y social.

Tabla 2: Participa en programas de rehabilitación.

\begin{tabular}{|c|c|c|c|c|}
\hline Alternativa & Frecuencia & Porcentaje & Porcentaje válido & Porcentaje acumulado \\
\hline Casi siempre & 37 & 18,7 & 18,7 & 18,7 \\
\hline Nunca & 152 & 76,8 & 76,8 & 95,5 \\
\hline Siempre & 9 & 4,5 & 4,5 & 100 \\
\hline Total & 198 & 100 & 100 & \\
\hline
\end{tabular}

La mayoría de los reclusos nunca han participado en programas de rehabilitación porque el CPLPACL Riobamba no dispone de un profesional del Trabajo Social para que desarrolle implemente este tipo de planes de intervención social que tanto beneficia al interno y su familia. 
Tabla 3: Recibe orientación psicosocial

\begin{tabular}{|c|c|c|c|c|}
\hline Alternativa & Frecuencia & Porcentaje & Porcentaje válido & Porcentaje acumulado \\
\hline Casi siempre & 56 & 28,3 & 28,3 & 28,3 \\
\hline Nunca & 142 & 71,7 & 71,7 & 100 \\
\hline Total & 198 & 100 & 100 & \\
\hline
\end{tabular}

La mayoría de los reclusos nunca han recibido orientación psicosocial por la falta de un equipo técnico multidisciplinario a través de los cuales puedan ser tratado de problemas que aquejan a los internos y que puedan ser estos, las causas por la que se encuentran en este centro. Por lo tanto, existe un riesgo mayor de reincidencia.

Tabla 4: Frecuencia de recepción de visitas

\begin{tabular}{|c|c|c|c|c|}
\hline Alternativa & Frecuencia & Porcentaje & Porcentaje válido & Porcentaje acumulado \\
\hline Casi siempre & 112 & 56,6 & 56,6 & 56,6 \\
\hline Nunca & 73 & 36,6 & 36,9 & 93,5 \\
\hline Siempre & 13 & 6,6 & 6,6 & 100 \\
\hline Total & 198 & 100 & 100 & \\
\hline
\end{tabular}

Los resultados demuestran que los PPLs CPLPACL Riobamba no reciben con frecuencia visitas de sus familiares lo cual afecta su autoestima, sus ganas de recuperar su vida y familia, de reconocer errores para cambiar y mejorar su estilo de vida, siendo este uno de los factores de reincidencia.

Tabla 5: Tipo de relación con su familia.

\begin{tabular}{|c|c|c|c|c|}
\hline Alternativa & Frecuencia & Porcentaje & Porcentaje válido & Porcentaje acumulado \\
\hline Buena & 196 & 99 & 99 & 99 \\
\hline Mala & 1 & 0,5 & 0,5 & 99,5 \\
\hline Muy buen & 1 & 0,5 & 0,5 & 100 \\
\hline Total & 198 & 100 & 100 & \\
\hline
\end{tabular}

El tipo de relación que mantienen los PPLs es muy importante para lograr procesos de reinserción y disminuir la reincidencia; los resultados obtenidos evidencian que es buen la relación con sus familias; en el proceso de entrevista se pudo conocer que la mayoría de los miembros del sistema familiar del interno desconocen que se encuentra privado de la libertad por esa razón no los visitan, pero si tienen buenos vínculos familiares.

Tabla 6: Se encuentra en proceso de reinserción familiar

\begin{tabular}{|c|c|c|c|c|}
\hline Alternativa & Frecuencia & Porcentaje & Porcentaje válido & Porcentaje acumulado \\
\hline No & 190 & 96 & 96 & 96 \\
\hline Si & 8 & 4 & 4 & 100 \\
\hline Total & 198 & 100 & 100 & \\
\hline
\end{tabular}


La mayoría de los reclusos señala que no forma parte de ningún programa de reinserción familiar porque no existe el profesional de Trabajo Social que oriente este tipo de procesos muy necesarios que permitan establecer herramientas y mecanismos capaces de generar perspectivas que posibilite una vida digna después de haber cumplido la condena. Por lo que la intervención del Trabajador Social es primordial en estos centros de privación de la libertad.

Tabla 7: Es visitado por amigos.

\begin{tabular}{|c|c|c|c|c|}
\hline Alternativa & Frecuencia & Porcentaje & Porcentaje válido & Porcentaje acumulado \\
\hline No & 169 & 85,4 & 85,4 & 85,4 \\
\hline Si & 29 & 14,6 & 14,6 & 100 \\
\hline Total & 198 & 100 & 100 & \\
\hline
\end{tabular}

La mayoría de los reclusos manifiestan que no son visitados por sus amigos, debido principalmente a que desconocen que se encuentran privados de la libertad o porque los rechazan al enterarse de la situación legal, otra de las causas es que no avisan porque sienten vergüenza y temor a sentir el rechazo de la sociedad.

Tabla 8: Familia tienen conocimiento donde se encuentra

\begin{tabular}{|c|c|c|c|c|}
\hline Alternativa & Frecuencia & Porcentaje & Porcentaje válido & Porcentaje acumulado \\
\hline No & 74 & 37,1 & 37,1 & 37,1 \\
\hline Si & 124 & 62,9 & 62,9 & 100 \\
\hline Total & 198 & 100 & 100 & \\
\hline
\end{tabular}

La mayoría de los reclusos señalan que sus familias si conocen donde se encuentran pero que igual casi siempre les visitan, es decir no es permanente la presencia familiar, por esta razón no tienen el apoyo continuo lo cual dificulta los procesos de reinserción y el riesgo de reincidencia.

Tabla 9: Al cumplir sentencia, su familia lo espera

\begin{tabular}{|c|c|c|c|c|}
\hline Alternativa & Frecuencia & Porcentaje & Porcentaje válido & Porcentaje acumulado \\
\hline No & 63 & 31,8 & 31,8 & 31,8 \\
\hline Si & 135 & 68,2 & 68,2 & 100 \\
\hline Total & 198 & 100 & 100 & \\
\hline
\end{tabular}

La mayoría de los reclusos señalan que sus familiares si les esperan al salir de libertad lo cual es importante para que desarrollen ellos planes de reinserción; es importante trabajar en el porcentaje de internos a quienes no los esperan sus familias porque tienden a reincidir. 
Tabla 10: Tiene algún proyecto de vida

\begin{tabular}{|c|c|c|c|c|}
\hline Alternativa & Frecuencia & Porcentaje & Porcentaje válido & Porcentaje acumulado \\
\hline No & 183 & 92,4 & 92,4 & 92,4 \\
\hline Si & 15 & 7,6 & 7,6 & 100 \\
\hline Total & 198 & 100 & 100 & \\
\hline
\end{tabular}

Es preocupante la cantidad de reclusos que no tienen un proyecto de vida, porque corren el riesgo de reincidencia y de continuar delinquiendo al no haber participado en programas de rehabilitación social y familiar. Se debe tomar en cuenta que tienen un nivel de instrucción primaria por lo que se hace necesario la implementación de planes laborales en los cuales puedan incursionar luego de recuperar su libertad.

Tabla 11: Es usted reincidente

\begin{tabular}{|c|c|c|c|c|}
\hline Alternativa & Frecuencia & Porcentaje & Porcentaje válido & Porcentaje acumulado \\
\hline No & 186 & 93,9 & 93,9 & 93,9 \\
\hline Si & 12 & 6,1 & 6,1 & 100 \\
\hline Total & 198 & 100 & 100 & \\
\hline
\end{tabular}

La mayoría de PPLs no son reincidentes, un factor importante es su edad, son jóvenes adultos que ingresaron al centro de reclusión entre los años 2014 a 2017 razón por la cual, probablemente es la primera vez que se encuentran en prisión.

\section{Conclusiones.}

- El Trabajo Social en los centros penitenciarios no es conocido (Flores, 2016) y en el caso del Ecuador es minimizada su importancia, de ahí la razón por la que varios de estos centros carecen de profesionales en esta rama que presten atención y medien en la restauración de los vínculos familiares deteriorados o rotos para tratar de favorecer la reinserción social del interno. Trujillo (2014) es más enfático al señalar que en los centros carcelarios existe una falta de rehabilitación y reinserción social hacia las personas privadas de la libertad, constituyéndose más bien en lugares de perfeccionamiento delictivo y criminal.

- De acuerdo con los datos obtenidos en el presente estudio, el 94\% de los encuestados señalan que nunca han sido atendidos por un Trabajador Social debido a que el CPLPACL Riobamba carece de un experto en esta área, atendida por profesionales ajenos al trabajo social, que carecen de conocimientos y estrategias para orientar y ayudar en los procesos de rehabilitación social, familiar y orientación psicosocial, misma que en un $71 \%$ nunca es atendida.

- En este mismo sentido, Molina (2017) señala que al interior de los centros de reclusión, el área trabajo social no despeña funciones pertinentes a la profesión, ni se orienta bajo enfoques humanistas de derechos o de grupos de atención prioritario porque realizan actividades ajenas a su profesión lo cual evidencia la 
poca contribución a la rehabilitación social de los PPLs que recuperan su libertan y reinciden en algún delito.

- La reinserción familiar definidamente no se está dando en los centros penitenciarios, así lo señala el $96 \%$ de los encuestados al afirmar que no se encuentran en ningún proceso de vinculación donde se trabaje directamente con el núcleo familiar del PPL. Esta situación significa una deuda del Estado según Peñafiel (2011), analista técnico del Ministerio de Justicia del Ecuador, quien admite que existen problemas por la falta de adecuados programas de capacitación, de estadística sobre expresidiarios reinsertados.

- Todas estas situaciones no hacen sino reprobar la política pública que el Estado viene aplicando en los centros penitenciarios, donde no se ha determinado metodología o proceso que garantice logar una rehabilitación social de las personas sentenciadas, peor aún se procura el bienestar del interno ni se dota de conocimientos y principios para facilitar la adaptación social al interior de estos centros para lograr una aceptable convivencia social.

- La falta de un proyecto de vida en un $92,4 \%$ abre camino a la reincidencia, estos datos se apoyan con las referencias que maneja el Ministerio de Justicia al señalar que en los últimos tres años el 9,4\% de la población carcelaria reingresaron a las cárceles del Ecuador.

- Es imprescindible educar al individuo para integrarlo a la sociedad y educar a la sociedad para que brinde nuevas oportunidades a este individuo, con el único fin de mejorar el círculo social del interno y comunitario; es decir de la sociedad en sí, que busca en el trabajo social la reinserción familiar efectiva del PPLs.

- En el CPLPACL Riobamba no se realizan actividades de intervención de trabajo social debido a la falta de profesionales especializados en esta área; la institución no dispone tampoco de equipos multidisciplinarios completos para que, de alguna forma, cubran esta carencia, por lo tanto, no se desarrollan programas de reinserción familiar ni social.

- Los factores que afectan los procesos de reinserción familiar del PACL en CPLPACLR Riobamba es la falta de profesional del Trabajo Social para que los oriente en procesos de reunificación familiar, social y específicamente legal.

- Se propone un plan de intervención de reinserción familiar para que sea presentado a las autoridades de la institución carcelaria a fin de que concienticen la necesidad de proveer al centro de reclusión de un profesional de Trabajo Social que lleve a efectos estos programas que tanto benefician a PPLs y familiares después de recuperar la libertad. 


\section{Referencias Bibliográficas}

Álvarez, A., Andrade, G., Arteaga, J., Barrera, E., Benalcázar, C., Coronel, A., . . . Serrano, E. (2014). Justicia. Obtenido de Manual de Derechos Humanos Aplicados al Contexto Penitenciario: https://www.justicia.gob.ec/wpcontent/uploads/downloads/2015/08/Manual-de-Derechos-Humanos-Aplicadosal-Contexto-Penitenciario.pdf

Flores, L. (2016). El Trabajo Social en los Centros Penitenciarios: Estudio realizado en la colonia penal de Islas Marías, México. Plataforma Académica de Trabajo Social, 15.

Molina, J. (2017). Estrategias de intervención del trabajo social con personas privadas de la libertad. Obtenido de http://dspace.ucuenca.edu.ec/bitstream/123456789/28662/1/Trabajo\%20de\%20T itulaci\%C3\%B3n.pdf

Peñafiel, G. (Enero de 2011). La Reinserción social de quien sale de prisión es una deuda del Estado. El Comercio, pág. 1.

Trujillo, D. (2014). Alternativas jurídicas para viabilizar la rehabilitación y reinserción social de las personas privadas de la libertad dentro de los centros carcelarios. Universidad de las Américas, 96.

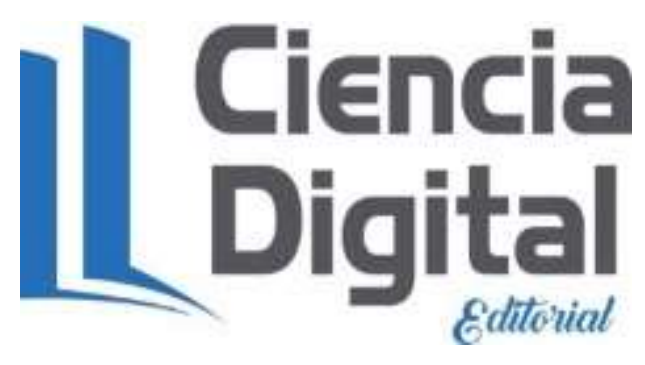




\section{Para citar el artículo indexado.}

Altamirano S. (2019). Rehabilitación y Trabajo Social en centros de privación de libertad en Ecuador. Revista electrónica Ciencia Digital 3(2), 172-180. Recuperado desde: http://cienciadigital.org/revistacienciadigital2/index.php/CienciaDigital/article/view/383 $\underline{1831}$

\section{Liencia}

El artículo que se publica es de exclusiva responsabilidad de los autores y no necesariamente reflejan el pensamiento de la Revista Ciencia Digital.

El artículo queda en propiedad de la revista y, por tanto, su publicación parcial y/o total en otro medio tiene que ser autorizado por el director de la Revista Ciencia Digital.
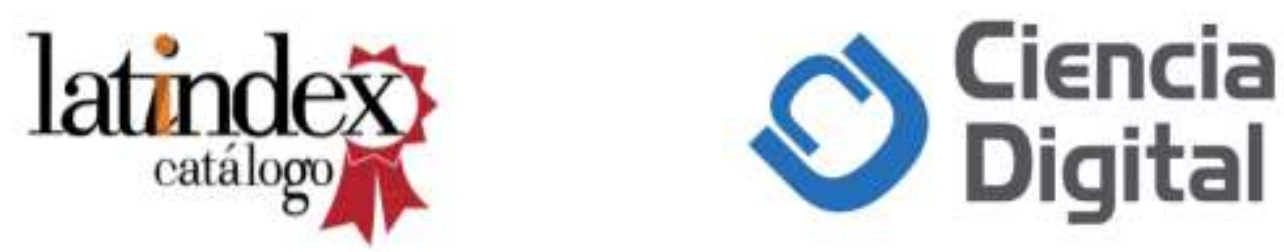